\title{
Die Top 100 Management Tools
}

Im Folgenden werden die 100 wichtigsten Konzepte, Methoden, Modelle und Instrumente - die Kernelemente der Management-Toolbox - in alphabetischer Reihenfolge dargestellt.

Um die Einbindung in einen anwendungsorientierten Kontext zu erleichtern, wird bei der Beschreibung der 100 Elemente der Management-Toolbox jeweils rechts vom Titel die Obergruppe symbolisiert und unterhalb des Titels die Untergruppe genannt.

Die Beschreibung der Konzepte, Methoden, Modellen und Instrumente folgt immer der gleichen Struktur: Die Kurzbeschreibung soll dem schnellen inhaltlichen Verständnis des Tools dienen. In welcher Form eine Anwendung erfolgen kann wird unter 2. skizziert. Der sich durch den Einsatz ergebende Nutzen sowie die für die Anwendung zu beachtenden Bedingungen sind Inhalt von 3. „Nutzen“ und 4. „Voraussetzungen“. Wichtige Elemente, Kernfragen, Vorgehensmodelle sind unter 5. „Kernelemente/Vorgehen“ beschrieben. Für weitergehende Fragen kann auf die unter 6. angegebenen Quellen bzw. weiterführende Literatur zurückgegriffen werden. 